\title{
Trabajando la coeducación a través del cuento de «La Cenicienta» desde un proceso de investigación-acción
}

\author{
Teaching co-education through the 'Cinderella' story, by means of a research-action \\ process
}

\author{
Desamparados Broch Martin (iD \\ e-mail: al286870@uji.es \\ Universitat Jaume I. España
}

Aida Sanahuja Ribés ID
e-mail: asanahuj@uji.es
Universitat Jaume I. España

Resumen

En este estudio partimos de lo que se entiende por coeducación y se hace un breve repaso sobre los principales hitos educativos acontecidos en referencia a dicho principio. En el marco teórico también se menciona el valor de los cuentos clásicos, en tanto y cuanto se convierten en un buen recurso para trabajar la coeducación. El objetivo principal de este estudio radica en planificar y poner en práctica actividades para trabajar la coeducación con el alumnado de infantil (a través de un proceso de investigación- acción), mediante el análisis y la reelaboración del cuento de La Cenicienta y las diferentes actividades derivadas del cuento. El proyecto se divide en diez sesiones, en las cuales se ha utilizado como eje central la literatura infantil para trabajar distintos estereotipos de género que se transmiten en el cuento y muchos siguen presentes en la sociedad actual. Con esto se busca que los estereotipos que se encuentran tan asimilados en la actualidad vayan cambiando y, por lo tanto, educar a las niñas y niños desde una perspectiva más inclusiva e igualitaria. Esta experiencia se ha llevado a cabo en un aula de infantil de cuatro años (en un centro de la provincia de Castellón) durante dos semanas del curso académico 2017-2018. Se ha obtenido un resultado óptimo que se refleja en las diferentes evidencias recogidas durante el proyecto. A modo de conclusión resaltar el potencial de los cuentos clásicos para trabajar valores en la etapa de educación infantil.

Palabras clave: coeducación; educación infantil; estereotipo de género; igualdad; literatura infantil

\begin{abstract}
In this study, we begin by defining what is understood by co-education and then we offer a brief review of the most important educational milestones achieved regarding co-education. The theoretical framework also mentions the value of classic stories, since they become a good resource for teaching co-education. The main objective of this study is to plan and implement activities to work co-education with preschool students (through a research-action process), by means of the analysis and reprocessing of the Cinderella story, and the different activities derived from this story. The Project is divided into ten sessions, in which children's literature has been used as a central axis to work on the different gender stereotypes that are transmitted in the story, and many of which are still present in today's society. With this, it is sought that stereotypes that are so assimilated nowadays start to change and thus, educate both girls and boys from a more inclusive and egalitarian perspective. This experience has been conducted in a 4-years old preschool classroom (in a school in the Province of Castellón) during two weeks in the academic year 2017-2018. An optimal result has been achieved that is reflected in the evidence collected during the Project. In conclusion, it is important to highlight the potential of classic stories to teach values in the stage of Childhood Education.
\end{abstract}

Keywords: coeducation; childhood education; gender stereotype; equality; children's literature

Recibido / Received: 13-12-2018

Aceptado / Accepted: 02-04-2019

Publicación en línea / Published online: 15-05-2019

Cómo referenciar este artículo / How to reference this article:

Broch Martin, D. \& Sanahuja Ribés, A. (2019). Trabajando la coeducación a través del cuento de «La Cenicienta» desde un proceso de investigación- acción. Tendencias Pedagógicas, 34, pp. 169-182. doi: $10.15366 / \mathrm{tp} 2019.34 .013$ 


\section{Introducción}

Las niñas y niños aprenden normas sociales de acuerdo con las costumbres y la cultura de cada país o región en la que viven, pero la sociedad se transforma y se adapta a los cambios sociales que surgen, aunque muchas veces, dichos cambios tardan en perpetuarse, dado que están muy arraigados. Como es el caso de la equidad o igualdad de género.

No obstante, consideramos la educación como una potente fuente para avanzar hacia una igualdad real, educando a personas críticas, libres, empáticas, tolerantes y respetuosas que sepan hacer frente a las demandas de la sociedad actual y estén preparadas para el futuro.

Así pues, se considera altamente importante y necesario que nuestras alumnas y alumnos no solo se eduquen de manera coeducativa, sino que interioricen los principios de lo que significa realmente la coeducación, para así, poder transformar y vivir en una sociedad más justa y equitativa.

\subsection{Pero..., ¿qué se entiende por coeducar?}

Siguiendo la definición propuesta por la RAE (Real Academia Española), coeducar significa: «Enseñar en una misma aula y con un mismo sistema educativo a alumnos de uno y otro sexo».

Tal y como se apunta des del Instituto Andaluz de la Mujer (IAM), coeducar no solo significa poner a alumnos de uno y otro sexo en la misma aula y con un mismo sistema educativo. Sino que coeducar va más allá. Dado que consiste en desarrollar todas las capacidades, tanto de los niños como de las niñas, a través de la educación. Supone eliminar estereotipos o ideas preconcebidas sobre las características que deben tener los niños y las niñas, los chicos y las chicas, los hombres y las mujeres.

Un estudio impulsado por Bian, Leslie y Cimpian (2017) demuestra como los estereotipos comunes (brillantez, genio, etc.) se asocian más con los hombres que con las mujeres. El estudio rebela que dichos estereotipos están respaldados e influyen en los intereses de los niños de 6 años. Estos hallazgos sugieren que las nociones sobre el género se adquieren de manera temprana y tienen un efecto inmediato en los intereses de los niños.

Por otro lado, Subirats y Tomé, explican las diferencias y presunciones que debe admitir un niño o niña desde que nace, afirmando que:

«Muchos de los mensajes que recibe la niña desde que nace van dirigidos a decirle que tiene que ser guapa, dulce, dócil; que es débil y que ha de evitar los peligros, no que tiene que ser fuerte y decidir por su cuenta». (2007, p. 49)

Cada niño o cada niña tiene derecho a ser diferente, por lo que es necesario educar valorando las diferencias individuales y las cualidades personales. Coeducar, tiene como objetivo hacer personas dialogantes e implica respeto y tolerancia, por lo que es una garantía para la prevención de la violencia. Mujeres en Red, define la coeducación como:

«El método de intervención educativo que va más allá de la educación mixta y cuyas bases se asientan en el reconocimiento de las potencialidades e individualidades de niñas y niños, independientemente de su sexo. Coeducar significa por tanto educar desde la igualdad de valores de las personas». (Mujeres en Red, El periódico feminista, 2006, p. 2)

Así pues, por todo lo expuesto anteriormente, coeducar significa preparar para la vida a personas, indistintamente de su sexo biológico o su identidad de género.

\subsection{Hacia la coeducación: un repaso sobre los principales hitos educativos}

Se considera oportuno realizar un breve repaso sobre los principales cambios que han acontecido en el sistema educativo desde que la figura de la mujer se incluyó en la enseñanza pública.

En el Informe Quintana (septiembre de 1813) se propone una educación pública y universal, aunque tras dicho informe, las niñas seguían excluidas. Más tarde, El Reglamento General de la Instrucción Pública (en 1821), acepta el proyecto de ley elaborado a partir del Informe Quintana. En el que la instrucción se dividía en pública y privada y la estructura educativa se fragmentaba en tres niveles: 
1) enseñanza; dirigida a la infancia, a leer, escribir, calcular y catecismo, 2) dirigida a la preparación de estudios superiores y 3 ) estudios superiores.

Es en 1857, con la Ley General de Instrucción Pública (más conocida como Ley Moyano), cuando se reconoce el derecho de las niñas a la educación y se intenta mejorar la tasa de alfabetismo en España. Cortada (1999) argumenta que, durante las siguientes décadas, las chicas se van incorporando progresivamente a la enseñanza, aunque con escuelas segregadas y currículos diferentes para ambos sexos (single-sex), donde las niñas estudiaban costura, cuidados de la casa, rezos, etc. Y los niños estudiaban escritura, lectura, cálculo, etc.

En palabras de González (2004), ya en el siglo XX, pedagogos y librepensadores como Francisco Ferrer y Guardia se acercaron a la idea de coeducación con la creación de la Escuela Moderna (mixta, laica y racionalista). Además, surgieron leyes que apoyaban la convivencia de ambos sexos en la escuela, como la Ley del 23 de junio de 1909, que implementaba la obligación de la enseñanza primaria hasta los doce años, o Dos Reales Órdenes de 8 de marzo y 2 de Septiembre donde se reconocía el derecho de las mujeres a la matriculación en la enseñanza oficial.

Según indica Cortada (1999), con la proclamación de la Segunda República, maestras como María Sánchez Arbós, Leonor Serrano o Margarita Comas, defendieron un modelo de coeducación en las escuelas que se asimilara al de los hombres, ya que el papel de la mujer iba cambiando en la sociedad. Se crearon muchas escuelas y se incrementó el índice femenino en la educación. Igualmente, este mismo autor afirma que con la dictadura franquista, todo lo que se había conseguido hasta ese punto se retrocedió con la prohibición de la escuela mixta en España. Por lo tanto, se volvió a la segregación en las aulas y a la escuela tradicional.

No es hasta el 1970, con La Ley General de Educación, cuando se anula la prohibición de escuela mixta y las asignaturas dirigidas específicamente a las mujeres desaparecen. El modelo de escuela mixta, no conllevaba la supresión del sexismo, por lo tanto, no existía una coeducación plena. Las mujeres, aunque fueron incluidas en el sistema educativo aún seguían siendo sometidas al machismo de la época.

A medida que se avanza en el tiempo, cada vez son más las mujeres que luchan por una igualdad social y educativa.

Es el 3 de octubre de 1990, cuando se instaura la Ley Orgánica de Ordenación General del Sistema Educativo (LOGSE), ley caracterizada por su currículo abierto y flexible. La cual establece como uno de los objetivos principales la no discriminación por razón de sexo tal como se recoge en el BOE (Boletín Oficial del Estado. Núm. 238, 1990) desde el Preámbulo:

«La educación permite, avanzar en la lucha contra la discriminación. La desigualdad, sean éstas por razón de nacimiento, raza, sexo, religión u opinión, tengan un origen familiar o social, se arrastren tradicionalmente o aparezcan continuamente con la dinámica de la sociedad».

Afirmándose en el Título Preliminar (BOE. Núm. 238, 1990, Artículo 2.3.C) «La efectiva igualdad de derechos entre los sexos, el rechazo de todo tipo de discriminación, y el respeto a todas las culturas».

La LOGSE fue más allá que las leyes anteriores, ofreciendo un currículo que se pudiera adaptar a las necesidades de cada centro e incorporando metodologías que potenciaran la igualdad de sexos de manera transversal.

Años más tarde, la LOGSE fue derogada por la LOE (Ley Orgánica de Educación 2/2006), la cual daba más importancia a la coeducación, aunque esta no supuso la implementación en las aulas de dichas proclamaciones por la igualdad.

En el año 2013 se aprobó la LOMCE (Ley Orgánica para la Mejora de la Calidad Educativa 8/2013). De esta última ley conviene destacar que uno de sus objetivos se centra en fomentar la igualdad efectiva de oportunidades entre hombres y mujeres. La ley apuesta por un sistema educativo de calidad, inclusivo, integrador, exigente y garantiza la igualdad de oportunidades.

Por añadidura, en la Comunidad Valenciana, la «Conselleria d'educació» está apostando fuerte porque la coeducación sea una realidad educativa, y por ello, hay nuevas normativas y protocolos (Instrucción 10425, 2016) para afrontar problemas como la identidad sexual y social. Asimismo, se ha creado la figura del Coordinador de Igualdad y Convivencia (Resolución 5291, 2016) en los centros educativos no universitarios. Además, se está elaborando toda una red de formación del profesorado con la finalidad de formar a los docentes en temas tan importantes como la inclusión educativa, la coeducación, prácticas innovadoras o plan para la coeducación. 


\subsection{Los cuentos clásicos, un buen recurso para trabajar la coeducación}

Una buena herramienta para que el alumnado interiorice los valores imperantes en la sociedad en la que vivimos radica en los cuentos clásicos:

«Las reivindicaciones que desde las diferentes corrientes se han manifestado en cuanto a lo que es adecuado o no, en sintonía con los valores emergentes de la sociedad actual, se han materializado en la reescritura de muchos de los cuentos clásicos. Surgen nuevas versiones que actualizando sus contenidos (tanto conceptuales como actitudinales), te ofrecen una visión moderna de los personajes de dichos cuentos e introducen temas objeto de reflexión y preocupación en la actualidad». (Salmerón, 2004, p. 87)

Por ende, la literatura infantil se convierte en uno de los mejores recursos de los que podemos disponer los docentes. Según Marín-Díaz y Sánchez-Cuenca (2015) los cuentos son breves narraciones directas y sin sucesos que distraen la atención de los niños, dado que solo se describe lo indispensable. Por lo tanto, es fácil para los menores seguir el hilo de la historia e interiorizar los contenidos que les queremos transmitir. Así pues, tenemos la gran oportunidad de transferir valores, tal como explica Roig (2014, p. 7) en su investigación:

«Los cuentos son una importante vía de transmisión y de expresión que permite a niños y niñas comprender el mundo, socializarse, aprender valores... por lo que los ámbitos educativos deben aprovecharlo para ofrecer alternativas y opciones respecto a valores, modelos y actitudes de acuerdo con la coeducación».

Además, la literatura infantil contribuye, en palabras de González (2007), a potenciar la atención, la memoria, la creatividad, la escucha activa, la comprensión verbal, la concentración, la imaginación y un sinfín más de aspectos tan necesarios en la formación del alumno. También ayuda a formar la conciencia del alumnado, tanto en el sentido moral, como en el cognitivo y el afectivo.

Adentrándonos en los cuentos clásicos, Vintró (2008, p. 21) también incide en la formación de valores y roles sociales. Diferentes cuentos clásicos como:

«La Cenicienta, Blancanieves y los siete enanitos o La Bella Durmiente del bosque, emiten un mensaje implícito: las mujeres o son buenas, sumisas y obedientes como sus protagonistas, o crueles y perversas, y reciben castigos por ello. En cambio, los príncipes son los que "despiertan" a las princesas dormidas, los que recorren "peligrosos" caminos, "vencen" a difíciles enemigos y los que "salvan" y "rescatan" a las doncellas».

Es decir, desde pequeños, se van interiorizando estas ideas y sin ser conscientes de ello, ciertos estereotipos de género se van marcando dentro de cada individuo. Y es irremediable no adquirir estas ideas en nuestra mente ya que es habitual poner la tele y que no aparezca una película de Disney recreando cualquiera de los cuentos de Charles Perrault o los hermanos Grimm.

Por lo tanto, es importante trabajar este tipo de materiales desde otra perspectiva, mostrarles a los menores la otra visión para que ellos, de forma crítica, puedan elegir que leer o de qué forma hacerlo.

«No se pretende desterrar de nuestras bibliotecas los cuentos o las historias que hasta ahora han cumplido su papel "educativo" de una brillante y divertida manera, más bien se reflexiona sobre la visibilización de los roles de género transmitidos, con el objetivo de introducir un punto de vista crítico hacia el material utilizado por la familia y la escuela, principales agentes socializadores». (García, 2013, p. 330)

Es decir, como afirma Vintró (2008, p. 7): «Se trata de no dar las cosas por supuestas, de no seguir aceptándolo todo porque es lo considerado "normal", si creemos que no es justo ni razonable». 


\section{Método}

Nos encontramos ante un proyecto que sigue una metodología de investigación-acción.

Así pues, en este trabajo se presenta un estudio de caso (Rodríguez Rojo, 2012; Simons, 2011; Stake, 1998) centrado en un proceso de investigación-acción (Latorre, 2003; Martínez, 2000; Contreras, 1994; Ander-Egg, 1990) articulado con el objetivo de implementar un proyecto basado en el trabajo de la coeducación en un aula de educación infantil a través del cuento de La Cenicienta. Conviene recordar que la investigación-acción nos permite indagar y reflexionar sobre la propia práctica profesional con el propósito de mejorarla (Latorre, 2003). Así pues, la investigación-acción resulta una estrategia eficaz para problematizar sobre la práctica con el fin de reorientarla (Moliner, Sanahuja y Benet, 2017).

\section{Contextualización y participantes}

Este proyecto se ha llevado a cabo en una escuela de la localidad de Nules (Castellón, España). Según el INE (Instituto Nacional de Estadística), a fecha de 2017, este municipio cuenta con 13.266 habitantes. Se localizan tres escuelas infantiles privadas y una pública, cinco CEIPs públicos y uno concertado y también un Instituto de Educación Secundaria y Formación Profesional.

La economía del pueblo se basa principalmente en la agricultura, con el predominio de cítricos. Y el nivel socioeconómico de los alumnos del centro donde se ha llevado a cabo el proyecto es medio, puesto que la mayoría de las familias se dedican al sector terciario (servicios).

Este proyecto se ha llevado a cabo en un aula de infantil cuatro años. Como se trata de un caso real, no se revelará ningún dato, reservando así el anonimato de los participantes ${ }^{1}$. La clase está compuesta por 13 alumnas y 8 alumnos, las características de los integrantes del aula son las acordes a la edad.

\section{Detección de las necesidades}

En el inicio del proceso de investigación-acción se utilizaron una serie de técnicas y herramientas, las cuales ayudaron a realizar un diagnóstico situacional. Seguidamente, se explican de manera breve cada una de ellas: a) análisis documental, b) diario de campo y c) actividad sobre conocimientos previos.

\subsection{Análisis documental}

Conviene apuntar que el análisis documental consiste en indagar sobre diferentes documentos o materiales que son objeto de estudio (Ruiz Olabuénaga, 2012; Álvarez, 2008; Tójar, 2006).

En primer lugar, se revisaron los principales documentos oficiales del centro, siendo estos: el Proyecto Educativo del Centro (PEC), el Reglamento de Régimen Interno (RRI), el Proyecto Curricular y el Plan de Convivencia. Se rastreó cada documento con el fin de conocer que aspectos se contemplaban en referencia a la coeducación.

De esta manera, se pudo comprobar como en el Proyecto Curricular del Centro, existía un apartado específico referente a los principios metodológicos. Específicamente, el punto «3.4. COEDUCACIÓN»:

«El colegio propugna una educación sin diferencias por razones de sexo y por lo tanto conseguir la igualdad de los y las alumnas y erradicar todo tipo de discriminación, evitando potenciar los roles tradicionales de hombres y mujeres [AM_PEC 2$]$ ».

\footnotetext{
${ }^{1}$ En cumplimiento del Reglamento 2016/679 del Parlamento Europeo y del Consejo de 27 de abril de 2016, referente a la protección de las personas físicas en cuanto al tratamiento de datos personales y a la libre circulación de estos datos, y de la Ley Orgánica 15/1999 de Protección de Datos de Carácter Personal.

2 AM: Análisis Material / PEC: Proyecto Curricular del Centro.
} 


\subsection{Diario de campo}

Además, se utilizó un diario de campo durante todo el proceso. Según afirma Albertín (2007, p. 14) el diario de campo permite: «registrar aquellos acontecimientos que transcurren en el día a día de su experiencia profesional o académica y que le resultan especialmente significativos».

En el diario de campo se anotaron comentarios o sucesos significativos que ocurrieron en el aula y que hacían ver que no se trabajaba la coeducación. Un ejemplo fue: «Se celebra un cumpleaños en clase, hay dos coronas, una rosa y una azul, la maestra le dice al niño que para él la azul que la rosa es de chicas» [DC $\left.{ }^{3}\right]$.

Así pues, en este instrumento se anotaba todo aquello que había acontecido y se creía interesante para realizar un diagnóstico lo más exhaustivo posible de las necesidades en el aula de infantil. Se pudo comprobar que, pese a estar establecido en el Proyecto Educativo de Centro, durante el proceso de detección de necesidades no se trabajó de manera explícita la coeducación en el aula.

\subsection{Actividad sobre conocimientos previos}

Para completar la detección de necesidades y una vez visto que no se trabajaban los principios de la coeducación en el aula objeto de estudio se planteó una actividad para conocer los conocimientos previos del alumnado.

Para ello, se confeccionó una actividad previa. Se elaboró una ficha conformada por cuatro preguntas y respuestas de elección múltiple: 1) ¿qué disfraz le pondrías a una chica?, 2) ¿qué disfraz le pondrías a un chico?, 3) ¿qué acciones puede hacer una chica?, 4) ¿qué acciones puede hacer un chico? Conviene apuntar que las respuestas estaban representadas por cuatro dibujos que el alumnado debía rodear. Las preguntas fueron leídas por la docente en voz alta y los alumnos contestaron rodeando con un círculo el dibujo que ellos creyeran conveniente. Esta actividad fue anónima y solamente había que indicar si era chica o chico quién lo había rellenado.

En esta tarea se pudo comprobar que la gran mayoría de alumnos del aula objeto de estudio tenían asimilados algunos estereotipos de género como que un chico puede jugar a fútbol y una chica hacer la comida, en vez de que ambos son capaces de hacer las dos cosas.

Llegados a este punto y por todo lo que se ha expuesto se decidió trazar una propuesta de actuación y elaborar una serie de sesiones para poner en práctica diferentes actividades y trabajar la coeducación en el aula.

5. Propuesta de acción: «Actividades para trabajar la coeducación mediante el cuento de $L a$ Cenicienta»

\subsection{Planteamiento de los objetivos de la acción}

El objetivo principal de este trabajo radica en planificar y poner en práctica actividades para trabajar la coeducación con el alumnado de infantil mediante el análisis y la reelaboración del cuento de La Cenicienta y las diferentes actividades derivadas del mismo.

Para la consecución de este objetivo principal se plantean una serie de objetivos específicos a trabajar en el aula objeto de estudio:

1) Reconocer elementos que aparecen en el cuento de La Cenicienta y otros elementos de la vida cotidiana sin hacer distinción por género.

2) Identificar y explicar la falsedad de diferentes afirmaciones referentes a los estereotipos de género.

3) Reelaborar el cuento de La Cenicienta de manera cooperativa y reflejarlo mediante la expresión artística.

\footnotetext{
${ }^{3}$ DC: Diario de Campo.
} 


\subsection{Temporalización}

El proyecto se desarrolló a través de diez sesiones. Dichas sesiones se llevaron a cabo durante el segundo trimestre del curso académico 2017-2018. Concretamente, del 16 al 27 de abril de 2018 de $15 \mathrm{~h}$ a $16.30 \mathrm{~h}$.

\subsection{Desarrollo de las sesiones}

El eje principal de las sesiones giraba en torno a la reelaboración y reescritura del cuento original de La Cenicienta, por el alumnado de infantil, con el propósito de trabajar desde los principios de la coeducación en el aula.

A continuación, en la Tabla 1, se explican brevemente cada una de las actividades llevadas a cabo en las diferentes sesiones.

Tabla 1.

Resumen de las actividades realizadas en cada una de las sesiones

\section{$1^{a}$ sesión: TOMA DE CONTACTO}

- Asamblea inicial. Cuestiones previas sobre el cuento de La Cenicienta: «¿Alguien conoce el cuento de Cenicienta?, ¿De qué trata?, ¿Quién es la protagonista?, ¿Dónde vive?, ¿Cómo creéis que es la chica que aparece en la portada? ¿Es alegre, es amable, es lista, es miedosa, es valiente?, ¿Y el chico, es miedoso, es valiente, es listo, es trabajador...? ¿Cómo sabéis si no la/lo conocéis? Si aún no hemos contado el cuento....»

- Contar el cuento original.

- Actividad en el patio. La maestra dice una serie de verbos y si el alumnado cree que puede realizar dichas acciones, tiene que realizar la acción de forma simbólica. Si por el contrario creen que no puede, tienen que levantar la mano. Cuando esto ocurre, se para la actividad para reflexionar porque creen que no pueden, hasta llegar a la conclusión de que todos podemos hacer las mismas acciones sin distinción de género.

- Asamblea final: reflexionar sobre la sesión.

\section{$2^{a}$ sesión: ¿SE PUEDEN RELACIONAR ELEMENTOS CON CHICAS O CHICOS?}

- Contar el cuento de La Cenicienta de nuevo, pero esta vez haciendo partícipes al alumnado.

- A continuación, tienen que relacionar distintos elementos con el chico, la chica o ambos. Se les presentan unos dibujos de elementos que usamos diariamente en casa, tanto chicos como chicas y algunos de ellos aparecen en el cuento abordado, como, por ejemplo: delantal, periódico, escoba, color rosa, color azul, coche, caballo, disfraz de príncipe, disfraz de princesa, corona, balón de fútbol y muñeca. Y en otro lado, en el suelo, un dibujo de un chico y de una chica. Una vez todos los elementos están claros, se les pide que relacionen cada uno de los elementos anteriores con la chica, con el chico o en medio de ambos si creen que se puede relacionar con ambos. Se solicita al alumnado que argumente el porqué de sus asignaciones.

- Se reparten los papeles de los protagonistas del cuento de La Cenicienta y se hace una asamblea para hablar de cómo se sienten y si se puede cambiar el cuento para que ellos al igual que Cenicienta se sientan mejor.

\section{$3^{a}$ sesión: FALSAS AFIRMACIONES SOBRE LOS ESTEREOTIPOS DE GÉNERO}

- Se leen algunas de las siguientes afirmaciones y se comentan: «Los chicos no lloran. Las chicas son las que limpian en casa. Los chicos y las chicas pueden cocinar. Los chicos solo juegan con pelotas. Las chicas solo juegan con muñecos. Los chicos y las chicas pueden montar a caballo. Los chicos no pueden utilizar el color rosa porque es de chicas. Los chicos son más fuertes que las chicas. Las chicas son más listas que los chicos. Los chicos pueden tener amigas chicas.»

- Asamblea para hablar sobre las conclusiones extraídas después de la actividad.

- Propuestas para reelaborar el cuento de La Cenicienta. La maestra anima al alumnado a que lancen sus propias hipótesis. Se anotan todos aquellos cambios que surjan, entre todos se llega a un consenso para cambiar el cuento original.

\section{$4^{a}$ sesión: REELABORACIÓN DEL CUENTO}

- La maestra ya ha escrito todos los cambios que han ido surgiendo durante los días anteriores y vuelve a contar el cuento con los cambios acordados, siempre teniendo en cuenta escribirlo con un lenguaje apropiado a la edad de los alumnos.

- Se dividen las viñetas del cuento que se ha elaborado. Para finalizar, la maestra asigna a los alumnos en pequeños grupos, tantos como viñetas se hayan creado.

\section{$5^{a}$ sesión: ¡MANOS A LA OBRA!}

- Las familias entran en el aula y se les presenta el cuento de La Cenicienta que han reelaborado entre todos. Se dividen los familiares participantes en los subgrupos de alumnos formados en la sesión anterior.

- Plasmar mediante la expresión artística en el papel continuo la viñeta que se les ha asignado a cada grupo.

- Una vez finalizada la actividad, se les anima a que en casa, con sus familias, hagan lo mismo con un cuento que seleccionen y el lunes en la asamblea cuenten el resultado. 
- Se presentan las viñetas ilustradas en el mural elaborado con las familias.

- Cuando se termina, en la pizarra, se hace una lista con todos los personajes que aparecen para elegir qué alumno representará a cada personaje. Para ello es preciso tener en cuenta sus preferencias y las características de los personajes y los alumnos para que todos y todas se sientan cómodos con el papel que representarán. Es importante que todos los alumnos intervengan en la representación y transmitirles que todos los papeles son necesarios por igual para que la obra tenga sentido.

- Diseño del guión.

\section{$7^{a}$ sesión: EMPIEZAN LOS ENSAYOS}

- Para esta sesión la maestra prepara previamente el aula como un escenario con carteles en los que hay dibujados los diferentes espacios de los que trata la obra. Y se explica al alumnado donde se tienen que situar. Una vez colocados, se ensaya y la maestra va dando paso a los alumnos para que hablen en el momento que les corresponda.

\section{$8^{a}$ sesión: LUCES...}

- Taller con las familias para preparar escenarios y disfraces. Para esta sesión vuelven a acudir las familias al aula. Puesto que en esta sesión se preparan los disfraces y decorados de los escenarios del cuento. Para ello, se realiza un taller en el que las familias están invitadas a traer todos los materiales que puedan aportar y no usen, como, por ejemplo: telas, sábanas viejas... Además, se aportan todos los materiales que se dispongan en clase en el rincón de los disfraces y material fungible que se necesite.

\section{$9^{a}$ sesión: CÁMARA...}

- Ensayo con los disfraces y escenarios elaborados. Además, para transmitirles tranquilidad, se les comunica que la tutora está presente en todo momento narrando el cuento y si necesitan ayuda durante la representación se les brinda.

- Representación de la función de teatro. Se invita a todas las familias a que acudan a verlo y una vez finalizado se les agradece su ayuda y disposición a lo largo de todo el proyecto.

Fuente: elaboración propia

\section{Recogida de evidencias}

\subsection{Técnicas e instrumentos para la recogida de evidencias}

\subsubsection{Observación}

Como técnica de recogida de información, para poder evaluar la puesta en marcha y la ejecución del proyecto, se ha utilizado la observación. La observación científica hace referencia a un proceso de contemplación sistemático y minucioso de un determinado fenómeno educativo (Ruiz Olabuénaga, 2012). La observación se centró en elementos importantes de los diferentes sucesos o acontecimientos que ocurrieron en las distintas actividades que los niños y niñas realizaron. Atendiendo principalmente a: 1) la actitud del alumnado en referencia a los aspectos relacionados con la coeducación, 2) la participación y 3) la escucha activa y el respeto hacia las diferentes opiniones de los compañeros y compañeras. Asimismo, conviene apuntar que la observación directa permitió al docente obtener información de las características psicomotoras, cognitivas y afectivas de los niños y niñas en cada actividad que se llevó a cabo durante el proyecto.

\subsubsection{Diario de campo}

Tal y como ya se ha mencionado en el apartado correspondiente a la detección de las necesidades, el diario de campo fue un instrumento que se utilizó a lo largo de todo el proceso. En esta fase del proceso de investigación-acción se utilizó anotando todas aquellas situaciones o sucesos que ocurrían en el aula y cabía destacar. Como, por ejemplo: reacciones, opiniones, reflexiones y acciones que se daban en el momento de las diferentes sesiones.

\subsubsection{Escala de estimación}

También se utilizó una escala de estimación. Una vez terminada la sesión, en una tabla, se anotaba la frecuencia, intensidad o duración de las conductas o actitudes que se habían abordado en las diferentes actividades. Este instrumento permite obtener unos resultados más centrados en los objetivos formulados en el proyecto. Estas escalas se han utilizado por jornada diaria y los resultados que se obtuvieron fueron a nivel individual, esto es, por estudiante. 


\subsubsection{Actividad con el alumnado después de las sesiones}

Se volvió a realizar la actividad que se efectuó durante la detección de las necesidades (ya explicada en el apartado 4.3), para poder comprobar si había cambiado la percepción del alumnado respecto al inicio del proyecto sobre coeducación.

\subsubsection{Entrevista a la docente}

Según Ruiz Olabuénaga (2012), la entrevista es una técnica propia de la investigación cualitativa consistente en obtener información de primera mano, mediante una conversación con los agentes implicados. Específicamente, se realizó una entrevista semiestructurada, puesto que se formularon algunos contenidos preestablecidos, pero dejando abierta la posibilidad al entrevistado de comentar otros aspectos relevantes (Báez, 2009). Así pues, se realizó una entrevista a la tutora del aula como acompañante del proceso para evaluar la práctica docente, la adecuación de los materiales empleados en el proyecto y el funcionamiento de las actividades propuestas. Seguidamente en la tabla 2, se presentan algunos ejemplos de cuestiones que se formularon en la entrevista.

Tabla 2.

Preguntas de la entrevista a la maestra

\begin{tabular}{c}
\hline Ejemplos de preguntas de la entrevista a la maestra \\
$\qquad$ ¿Crees que los materiales han sido adecuados? \\
$¿$ Cómo crees que ha sido el funcionamiento de las actividades? \\
$¿$ Qué se puede mejorar? \\
$¿$ Cambiarías algo?
\end{tabular}

Fuente: elaboración propia.

Con todas las técnicas e instrumentos descritos se pretendía llevar a cabo la triangulación como estrategia en la investigación educativa que dota de calidad y rigurosidad a la investigación cualitativa (Aguilar y Barroso, 2015; Okuda y Gómez-Restrepo, 2005; Cisterna, 2005).

\subsection{Resultados}

Los resultados del estudio se presentan siguiendo el principal objetivo que ha guiado este trabajo centrado en planificar y poner en práctica actividades para trabajar la coeducación con el alumnado de infantil mediante el análisis y la reelaboración del cuento de La Cenicienta y las diferentes actividades derivadas del mismo.

En primer lugar, conviene subrayar que la planificación y la puesta en práctica de las actividades para trabajar la coeducación se ha efectuado mediante un proceso de investigación-acción. Seguidamente, se presenta en la Tabla 3 una síntesis de todo el proceso realizado.

Tabla 3.

Sintesis del proceso de investigación-acción

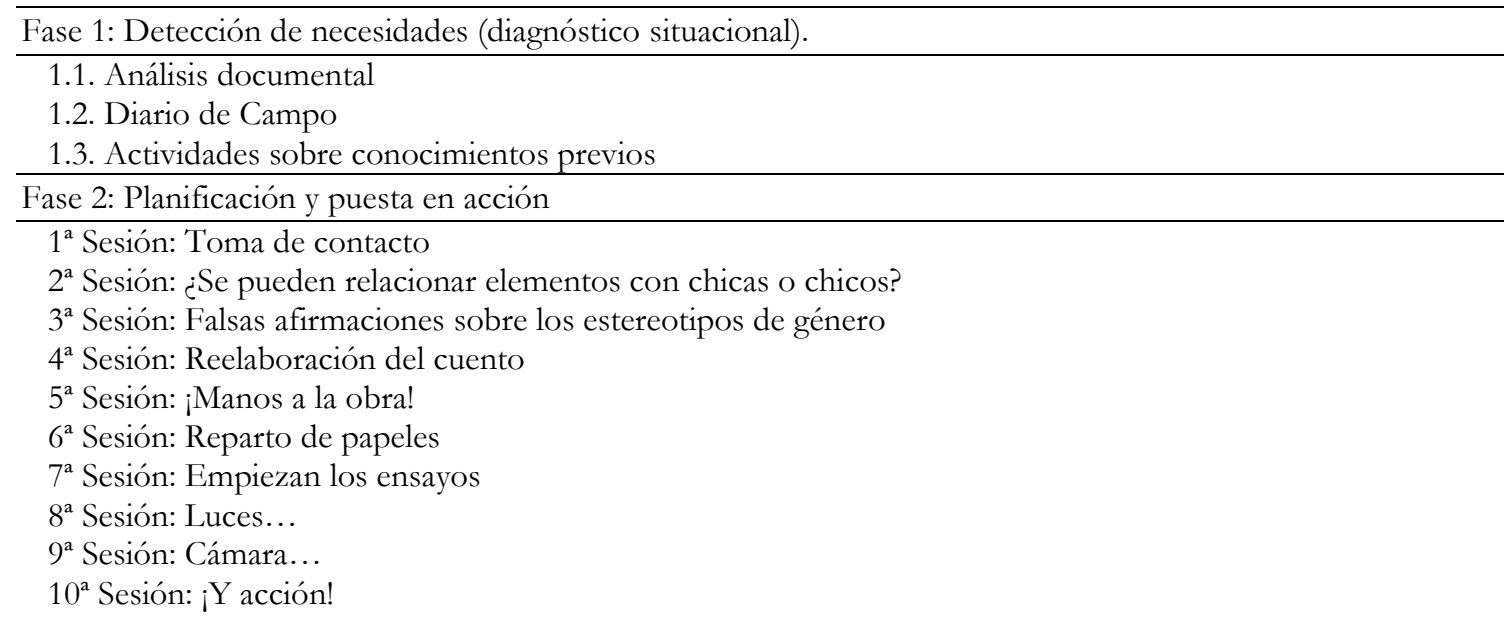




\begin{tabular}{l}
\hline Fase 3: Seguimiento del proceso y evaluación: recogida de evidencias \\
\hline Observación \\
Diario de campo \\
Escala de estimación \\
Actividad punto 1.3 (alumnado) \\
Entrevista a la docente \\
\hline Fase 4: Propuestas de mejora (nueva propuesta de acción) \\
\hline
\end{tabular}

Fuente: elaboración propia.

Pese a la corta edad de los participantes (alumnado de infantil 4 años), ya se detectó en las primeras sesiones del proyecto que los alumnos tenían arraigadas ciertas creencias y percepciones en relación al género femenino o masculino (objetivo específico 2. Identificar y explicar la falsedad de diferentes afirmaciones referentes a los estereotipos de género). Esto es, desde un principio se tuvo que dialogar y reflexionar sobre porqué creían que no podían realizarla ciertas actividades por ser chicos o por ser chicas, y llegar a la conclusión de que todas y todos podemos realizar cualquier acción. Con relación a esta idea que se viene desarrollando, en el diario de campo se recogieron algunas expresiones emitidas por el alumnado. Por ejemplo: «los tacones los llevan las chicas», «porque duelen los pies», «me podría caen» [DC]. Se reflexionó al respecto planteando la idea que a las chicas también les podían doler los pies al llevar tacones. Durante la tercera sesión se experimentaron grandes cambios porque desmintieron todas las afirmaciones falsas: «porque todos podemos hacer todo», «sí, porque los chicos somos iguales que las chicas, a todos nos gusta jugar a fútbol y nadie es más listo que otro» [DC].

En cuanto a la actividad planteada en la segunda sesión, mediante la observación directa, se pudo apreciar que el alumnado relacionaba los elementos por distinción de género, es decir, el color rosa lo relacionaron con la chica y el color azul con el chico (objetivo específico 1. Reconocer elementos que aparecen en el cuento de La Cenicienta y otros elementos de la vida cotidiana sin hacer distinción por género). Al finalizar la sesión, se les preguntó porque habían hecho esa relación y un niño contestó: «porque el rosa es de chicas» [DC] a lo que otro niño aportó: «pues a mí me gusta el rosa» [DC] y se creó un pequeño debate en el que reflexionaron en torno a lo que se había hablado el día anterior. Se transmitió que ningún color se corresponde con ningún género, para explicar que cada uno tenemos gustos diferentes.

Hay que destacar que la asamblea ha resultado una muy buena estrategia a lo largo del proceso para razonar sobre las conclusiones extraídas después de cada una de las actividades planteadas. Mediante la observación, se pudo ver que los alumnos participaban y se formaban pequeños debates. Por ejemplo: «Sí, todos podemos hacer todo», «Claro, yo puedo llevar tacones, aunque creo que me caería», "pues yo creo que no tendrían que llevar tacones las chicas, porque seguro que luego les duelen los pies», «En mi casa todos limpiamos cuando no hay que venir al cole», «pues en la mía mi padre siempre hace la comida y mi madre friega después de cenar» [DC].

Por lo que respecta a la participación del alumnado en las actividades planteadas en el proyecto fue in crescendo. Mediante la observación se pudo comprobar que la mayoría han participado activamente en todas las sesiones, aunque ha habido actividades en las que a algunos alumnos y alumnas les ha costado más o apenas han participado. Este aspecto se recogió con más detalle en la escala de estimación, y se apreció que solamente en una actividad dentro de una sesión un alumno no participó, por lo demás, ha habido sesiones en las que el grado de aportaciones ha sido más o menos el mismo, pero todos han colaborado conjuntamente durante el desarrollo de las sesiones. Así mismo, hay que subrayar que, durante la segunda sesión, a través de la observación directa se detectó que no todo el alumnado colaboró aportando ideas. En concreto, cinco alumnos no hablaban, por lo que se les incitó a proponer alguna sugerencia y de los cinco contestaban: «No sé qué cambiar», «No sé» [DC]. En la tercera sesión, se observó que los alumnos que no habían aportado ideas el día anterior se mostraban más participativos. Todo el estudiantado habló cooperando entre ellos y aportando ideas para la reelaboración del cuento de La Cenicienta (objetivo específico 3. Reelaborar el cuento de La Cenicienta de manera cooperativa y reflejarlo mediante la expresión artística). De aquí se recogió en el diario de campo notas como: «Cenicienta puede no llevar tacones en la fiesta, porque los tacones hacen daño», «su madrastra puede ser buena y quererla mucho», «sus hermanas pueden ayudar a Cenicienta a elegir el vestido», «podría ir con chándal». Para la cuarta sesión, hicieron aportaciones como: «la princesa podía ser Cenicienta y el príncipe el que pierde el zapato», «Cenicienta podría tener un perro», «el príncipe se podría llamar Carlos», «La Cenicienta podríamos llamarla Carlota, porque mi prima se llama Carlota y me gusta mucho ese 
nombre», «este cuento es más bonito que el de La Cenicienta de verdad», «¿a qué ahora Carlota está más contenta que Cenicienta de antes?», «podríamos hacer esto con todos los cuentos» [DC], entre todos y todas se decidió qué cambios añadir al cuento y acabarlo de reelaborar.

En cuanto a la actividad que se realizó tanto al inicio (detección de las necesidades) como al finalizar el proyecto, se pudo comprobar que la mayoría de los alumnos cambiaron sus respuestas. En la primera pregunta, ¿qué disfraz le pondrías a una chica?, excepto dos casos (uno marcó la primera opción, y otro las dos últimas) todos marcaron las cuatro opciones. Es decir, la mayoría de los alumnos, tras las actividades realizadas, habían entendido que todos podemos vestir cualquier disfraz sin distinción por género. En la segunda pregunta, ¿qué disfraz le pondrías a un chico?, todos marcaron todas las opciones, salvo tres casos, que las marcaron todas menos la tercera alternativa, por lo que, al igual que la anterior, la mayoría del alumnado entendió que todos podemos vestir cualquier disfraz. Y en las dos últimas preguntas, ¿qué acciones puede hacer un chico? y ¿qué acciones puede hacer una chica? todo el alumnado marcó todas las opciones, dejando ver que, después de trabajar las actividades propuestas anteriormente, había habido un cambio respecto a los estereotipos de género que tenían tan marcados anteriormente.

De la entrevista con la tutora, se recogieron valoraciones muy positivas del proyecto: «el proyecto ha sido precioso, ojalá se trabajara más este tipo de actitudes en todas las aulas» [E]. Resaltar la idea de poder trabajar valores a través de la literatura infantil: «se ha visto lo importante que es el trabajo de los valores en el aulas [E]. Una de las principales propuestas de mejora que plantea la tutora reside en el tiempo destinado al proyecto: «igual lo hubiera alargado más visto el interés que tenían los alumnos en el tema» [E]. Así mismo, «no cambiaría ninguna actividad del proyecto» [E]. La docente considera que a través de estos proyectos se puede aportar un granito de arena para que la sociedad cambie: «somos capaces de ayudar a que la sociedad cambie» [E].

\section{Discusiones de los resultados y conclusiones}

Para concluir el trabajo, conviene apuntar que después de ver los resultados alcanzados en el proyecto, el objetivo principal que se ha formulado se ha cumplido satisfactoriamente.

Se ha visto como la coeducación va mucho más allá de la definición aportada por la RAE, dado que, coincidiendo con el Instituto Andaluz de la Mujer y Mujeres en Red, coeducar significa preparar para la vida a personas, indistintamente de su sexo biológico o su identidad de género. Ayudar a crear personas críticas, libres, empáticas, tolerantes, respetuosas, que sepan desenvolverse en sociedad y estén preparadas para el futuro.

Pese a que se ha avanzado mucho en esta temática, tal y como hemos podido apreciar en los diferentes hitos acontecidos (Cortada, 1999; González, 2004) y las diferentes legislaciones aportadas, todavía existe un largo camino por recorrer. Pese a la joven edad de los participantes en este estudio se han detectado ciertas actitudes y comentarios que apuntan a la asunción de diferentes roles a los varones $\mathrm{u}$ otros a las mujeres, por el simple hecho de serlo. Esto va en sintonía con los hallazgos de investigaciones como la de Bian, Leslie y Cimpian (2017) y con lo que explican Subirats y Tomé (2007).

Con la ejecución de este proyecto se ha podido contrastar que, efectivamente, los cuentos potencian un sinfín de aspectos como la creatividad, la atención, la escucha activa, la imaginación (Rodari, 2004) y la conciencia: tanto en el sentido moral, como en el cognitivo y el afectivo (González, 2007). Puesto que, a través de los cuentos se transmiten y expresan miles de ideas que se interiorizan sin darse cuenta (Roig, 2004), así como un sinfín de valores (Vintró, 2008). Por todo esto, es tan importante trabajar estos aspectos desde la infancia, ya que es durante este periodo cuando se aprenden estas normas sociales y se considera el cuento infantil como una potente herramienta socializadora (Ros, 2013). Es por ello que, podemos corroborar lo que ya han apuntado otros estudios (Agudo, 2017) considerando a los cuentos como una potente herramienta para introducir el trabajo en valores sobre todo en la etapa de educación infantil.

Conviene subrayar que no se trata de dejar a un lado los cuentos clásicos, sino que como explicaba Garcia (2013), se trata de trabajarlos, reflexionar sobre los estereotipos de género que nos transmiten e introducir una mirada crítica en el alumnado para que ellos sean conscientes de dichas injusticias y no den cosas por supuestas, como afirma Vintró (2008), que no sigan aceptando dichas cosas porque se consideren «normales». Así pues, se concibe de suma importancia incorporar aspectos relacionados con la coeducación tanto en la formación inicial como en la formación continua de los docentes, ya 
que en ocasiones el profesorado no cuenta con las estrategias o herramientas suficientes para trabajarlo en su aula (Ortega, 2019).

Remarcar que el hecho de haber implementado el proyecto siguiendo un proceso de investigaciónacción (Moliner, Sanahuja y Benet, 2017; Latorre, 2003; Martínez, 2000; Contreras, 1994; Ander-Egg, 1990), ha permitido partir de las necesidades del contexto y ajustar las actividades al mismo. Además, se ha suscitado un interés por continuar trabajando en esta línea.

Como principal propuesta de mejora al proyecto que aquí se ha presentado conviene destacar que se debería trabajar de manera transversal y a lo largo de todo el curso académico un proyecto que articule la coeducación como eje central. A menudo, por cuestiones de programación o currículo, se suelen hacer proyectos esporádicos para trabajar diferentes temáticas o cuestiones que se introducen de manera aislada sin tener un gran calado en el alumnado. Se propone alargar las sesiones visto el éxito y las ganas que tenía el alumnado de seguir con el tema tratado y empezar con otros libros para trabajarlos de igual manera introduciendo diferentes tipos de actividades. Por ende, consideramos importante compartir esta experiencia ya que se han detectado cambios en los comentarios y actitudes de los alumnos con relación a la temática abordada. Podemos decir que la coeducación no se debería trabajar de manera puntual en un proyecto, sino que se debería trabajar de manera integrada en el día a día del aula.

\section{Referencias}

Agudo, C. (2017). El trabajo en valores a través del cuento en el aula de Educación Infantil. (Trabajo Final de Grado). Universidad de Valladolid. Recuperado de: http://uvadoc.uva.es/handle/10324/29205

Aguilar, S. \& Barroso, J. (2015). La triangulación de datos como estrategia en investigación educativa. Revista de Medios y Educación, 47, pp. 73-88. doi: https://doi.org/10.12795/pixelbit.2015.i47.05

Albertín, P. (2007). La formación reflexiva como competencia profesional. Condiciones psicosociales para una práctica reflexiva. El diario de campo como herramienta. Revista de Enseñanza Universitaria, 30, pp. 7-18. Recuperado de: http://institucional.us.es/revistas/universitaria/30/Albertin.pdf

Álvarez, C. Á. (2008). La etnografía como modelo de investigación en educación. Gazeta de antropología, 24(1). Recuperado de: www.ugr.es/ pwlac/G24_10Carmen_Alvarez_Alvarez.html

Ander-Egg, E. (1990). Repensando la Investigación-Acción-Participativa, comentarios, críticas y sugerencias. Vitoria-Gasteiz: Dirección de Bienestar Social.

Báez, J. (2009). Investigación cualitativa. Madrid: ESIC.

Bian, L, Leslie, S-J., \& Cimpian, A. (2017). Gender stereotypes about intellectual ability emerge early and influence children's interests. Science, 355, pp. 389-391. doi: https://doi.org/10.1126/science.aah6524

Cisterna, F. (2005). Categorización y triangulación como procesos de validación del conocimiento en investigación cualitativa. Theoria, 14(1), pp. 61-71. Recuperado de: $<$ http://www.redalyc.org/articulo.oa?id $=29900107>$

Contreras, J. (1994). La investigación en la acción: ¿Qué es? Cuadernos de Pedagogía, 224, pp. 8-12.

Cortada, E. (1999). De las escuelas de niñas a las políticas de igualdad. La Educación, monográfico entre dos siglos. Cuadernos de Pedagogía, 286, pp. 43-47. Recuperado de: http://uom.uib.cat/digita1Assets/202/202207_14.pdf 
Decreto 38/2008, de 28 de marzo, del Consell, por el que se establece el currículo del segundo ciclo de la Educación Infantil en la Comunitat Valenciana. [2008/3838]. Recuperado de: http://www.dogv.gva.es/datos/2008/04/03/pdf/2008_3838.pdf

González, J. (2007). La argumentación a partir de cuentos infantiles. Revista Mexicana de Investigación Educativa, 12(33), pp. 657-677. Recuperado de: http:/ /www.redalyc.org/articulo.oa?id=14003310

González, T. (2004). Una apuesta por la educación de las mujeres o el discurso alternativo del anarquismo español. Historia Caribe, 9, pp. 95-106. Recuperado de: https://dialnet.unirioja.es/servlet/articulo?codigo $=2308223$

Instituto Andaluz de La Mujer (IAM) (2005). Coeducación. Sevilla: Consejería para la Igualdad y Bienestar Social.

Instrucción del 15 de diciembre de 2016, del director general de Política Educativa, por la que se establece el protocolo de acompañamiento para garantizar el derecho a la identidad de género, la expresión de género y la intersexualidad. [2016/10425]. Recuperado de: https://www.dogv.gva.es/datos/2016/12/27/pdf/2016_10425.pdf

Latorre, A. (2003). La investigación-acción. Conocer y cambiar la práctica educativa. Barcelona: Graó.

Ley Moyano, Informe Quintana, de 9 de septiembre, Cádiz. Recuperado de: http:/ /personal.us.es/alporu/historia/ley_moyano.htm

Ley Orgánica 1/1990, de 3 de octubre, de Ordenación General del Sistema Educativo. Boletín Oficial del Estado, 4 de octubre de 1990, núm. 238, pp. 28927-28942. Recuperado de: https://www.boe.es/boe/dias/1990/10/04/pdfs/A28927-28942.pdf

Ley Orgánica 2/2006, de 3 de mayo, de Educación. Boletín Oficial del Estado, 4 de mayo de 2006, núm. 106, pp. 17158-17207. Recuperado de: https://www.boe.es/buscar/pdf/2006/BOE-A-20067899-consolidado.pdf

Ley Orgánica 8/2013, de 9 de diciembre, para la mejora de la calidad educativa. Boletín Oficial del Estado, 10 de diciembre de 2013, núm. 295, pp. 97859-97921. Recuperado de: http://www.boe.es/boe/dias/2013/12//10/pdfs/BOE-A-2013-12886.pdf

Marín-Díaz, V. \& Sánchez-Cuenca, C. (2015). Formación en valores y cuentos tradicionales en la etapa de educación infantil. Revista Latinoamericana de Ciencias Sociales, Niñez y Juventud, 13(2), pp. 1093-1106. doi: https://doi.org/10.11600/1692715x.13238190514

Martínez, M. (2000). La investigación-acción en el aula. Agenda Académica, 7(1), pp. 27-39. Recuperado de: http:/ / files.docentia.webnode.es/200000031-e2181e310b/ia.pdf

Moliner, O., Sanahuja, A., \& Benet, A. (2017). Prácticas inclusivas en el aula desde la investigación-acción. Colección Sapientia 127. Castellón de la Plana, España: Servicio de Publicación de la Universitat Jaume I. doi: https://doi.org/10.6035/Sapientia127

Mujeres en Red, El periódico feminista, (2006), Recuperado de: http://www.mujeresenred.net/IMG/article_PDF/article_a1304.pdf

Okuda, M. \& Gómez-Restrepo, C., (2005). Métodos en investigación cualitativa: triangulación. Revista Colombiana de Psiquiatría, XXXIV(1), pp. 118-124. Recuperado de: http://www.scielo.org.co/scielo.php?script=sci_arttext\&pid=S0034-74502005000100008 
Ortega, M. R. (2019). La perspectiva de género: marco normativo y formación inicial y permanente de docentes en educación preescolar. Revista Latinoamericana de Educación y Estudios Interculturales, 3(1), pp. 71-77 Recuperado de: http://cresur.edu.mx/OJS/index.php/RLEEI_CRESUR/article/view/327/245

Real Academia Española. (2001). Disquisición. En Diccionario de la lengua española (22.a ed.). Recuperado de http://dle.rae.es/?id=9e42EIP

Resolución de 1 de julio de 2016, de las direcciones generales de Política Educativa y de Centros y Personal Docente, por la que se dictan instrucciones en materia de ordenación 25 académica y de organización de la actividad docente en los centros que impartan Educación Secundaria Obligatoria y Bachillerato durante el curso 2016-2017. [2016/5291] Recuperado de: http://www.dogv.gva.es/datos/2016/07/12/pdf/2016_5291.pdf

Rodari, G. (2004). La imaginación en la literatura infantil. Perspectiva Escolar, 43 Recuperado de: https://www.imaginaria.com.ar/12/5/rodari2.htm

Rodríguez Rojo, M. (2012). Cómo investigar en estudio de casos. Guía práctica. Boliva: CEDID-FIFIED.

Roig, C. A. (2014). Coeducación a través de los cuentos en los centros de segundo ciclo de Educación Infantil de Mallorca. (Trabajo Final de Grado). Universitat de les Illes Balears Recuperado de: http://dspace.uib.es/xmlui/bitstream/handle/11201/823/TFGCatianaRoig.pdf?sequence $=1 \&$ is Allowed $=\mathrm{y}$

Ros, E. (2013). El cuento infantil como herramienta socializadora de género. Cuestiones Pedagógicas, 22, pp. 330-350. Recuperado de: http://institucional.us.es/revistas/cuestiones/22/art_15.pdf

Ruiz Olabuénaga, J. I. (2012). Metodología de la investigación cualitativa. Bilbao, España: Deusto.

Salmerón, P. (2004). Transmisión de los cuentos a través de los cuentos clásicos infantiles. (Tesis doctoral). Universidad de Granada, Granada. Recuperado de: http://0-hera.ugr.es.adrastea.ugr.es/tesisugr/15487441.pdf

Sánchez Cuenca, C. (2016). Desarrollo de valores a través de los cuentos, con metodologías tradicionales o tics, en la etapa de educación infantil (Tesis doctoral). Universidad de Córdoba. Recuperado de: https://helvia.uco.es/bitstream/handle/10396/14800/2017000001568.pdf?sequence=1\&isAllowed=y

Simons, H. (2011). El estudio de caso: Teoría y práctica. Madrid: Morata.

Stake, R. E. (1998). Investigación con estudio de casos. Madrid: Morata.

Subirats, M. \& Tomé, A. (2007) Balones fuera. Reconstruir los espacios desde la coeducación. Barcelona: Octaedro.

Tójar, J. C. (2006). Investigación cualitativa comprender y actuar. Madrid: La Muralla.

Vintró, M. (2008). Sexismo en la literatura infantil. Cuadernos de Literatura Infantil y Juvenil, 217, pp. 21-27. Recuperado de: http://prensahistorica.mcu.es/es/catalogo_imagenes/grupo.cmd?path=1000125297 